\title{
Development of Hand Grip Assistive Device Control System for Old People through Electromyography (EMG) Signal Acquisitions
}

\author{
Herman Khamis ${ }^{1}$, Shahrol Mohamaddan ${ }^{1, a}$, Takashi Komeda ${ }^{2}$, Aidil Azli Alias ${ }^{1}$, Shirley Jonathan Tanjong ${ }^{1}$, Norhuzaimin \\ Julai $^{1}$ and Nurul 'Izzati Hashim ${ }^{1}$ \\ ${ }^{1}$ Faculty of Engineering, Universiti Malaysia Sarawak (UNIMAS), 94300 Kota Samarahan, Sarawak, Malaysia \\ ${ }^{2}$ Department of Bioscience and Engineering, College of System Engineering and Science, Shibaura Institute of Technology, 3308570 \\ Saitama, Japan
}

\begin{abstract}
The hand grip assistive device is a glove to assist old people who suffer from hand weakness in their daily life activities. The device earlier control system only use simple on and off switch. This required old people to use both hand to activate the device. The new control system of the hand grip assistive device was developed to allow single hand operation for old people. New control system take advantages of electromyography (EMG) and flex sensor which was implemented to the device. It was programmed into active and semi-active mode operation. EMG sensors were placed on the forearm to capture EMG signal of Flexor Digitorum Profundus muscle to activate the device. Flex sensor was used to indicate the finger position and placed on top of the finger. The signal from both sensors then used to control the device. The new control system allowed single hand operation and designed to prevent user from over depended on the device by activating it through moving their fingers.
\end{abstract}

\section{Introduction}

In most of developed world countries, it is accepted that the age of 65 years old is the age of old people while the United Nation (UN) agreed that 60 years old or more is an age that used to refer the older population [1]. There is no specific age range for old age. However, old age always refers to an age that close or exceed the life expectancy of human beings.

In current global population trend, it is shown that there is increasing numbers of old people from total population. Old people whose their age is more than 60 years toke up 9.2 percent of the global population in 1990 and it increased to 11.7 percent in 2013. By 2050, it is expected that the population of old people to increase to 21.1 percent [2]. The increasing numbers of old people population is due to the fact that currently, the world is experience the declining of birth rate from years to years. As in 1950, the crude birth rate (number of live birth per 1,000 people per year) is 37.0 and decreased to 20.6 in 2000 and expected to continue decrease to 13.4 in 2050 [3]. Besides that, the increasing of the life expectancy years in current population also contributed to the increasing of the old people population as in 2015, the global life expectancy from birth is 71.4 years and from 2000 to 2015, there is high increase of global average life expectancy by 5 years [4].

The increases of old people population will lead to the increase of demand in health care as old people are more susceptible to suffer diseases and syndromes. They also suffer from decrease of physical capabilities. These make

\footnotetext{
a Corresponding author: mshahrol@unimas.my
}

it difficult for old people to carry out daily life activities such as standing, walking, chores, exercises and many more. Hand is one of the important parts of human body that can be deteriorated in function capabilities due to ageing factor. Old people hand become weak and having difficulty to do basic movement of hand such as gripping, pinching, eating and wearing clothes. Therefore, to help old people to overcome this hand weakness problem and to assist them in their daily life activities, devices which help to amplify the hand strength is needed.

Several researches have been conducted in developing device that aims to amplify hand function. The device take form of hand exoskeleton which is not just limited for old people that suffer from hand weakness but also for other application that required to amplify the hand strength and rehabilitation. The hand exoskeleton can be classified into its purpose, power transmission mechanism, actuator and intention sensing method [5].

Hand exoskeleton purpose can be divided into two which are for hand rehabilitation [6-15] to regain the loss hand function due diseases such as stroke. Another purpose of hand exoskeleton is for assistance [16-19] which used to assist the hand function such as for hand weakness of old people.

Hand grip assistive device was also developed to provide assistant for old people whose suffer from hand weakness especially during gripping activities. The hand grip assistive device is shown in Figure 1.

The device use tendon driven mechanism $[12,16]$ as it power transmission mechanism to move the finger which allow the finger to flex. It was powered by electric motor 
$[6-12,16,19]$ to actuate the three fingers which are thumb, index and middle finger. Single rotary motor was used in the device to keep the minimum weight. The motor was attached with clutch gear system as shown in Figure 2. The purpose of clutch gear system is to allow the motor to pull the driven cable and also free up the cable. However, the control system for this device is quite lacking as user need to push button manually to activates it.

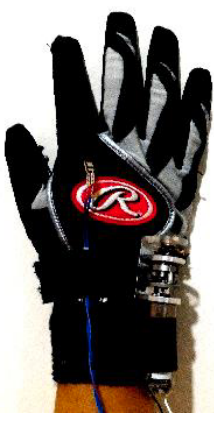

(a)

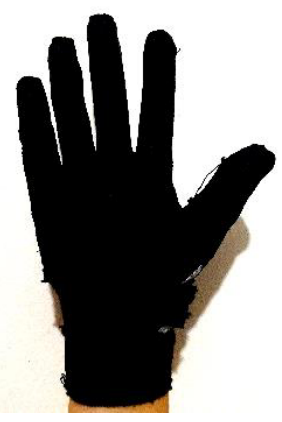

(b)
Figure 1. Hand grip assistive device (a) back view (b) front view.

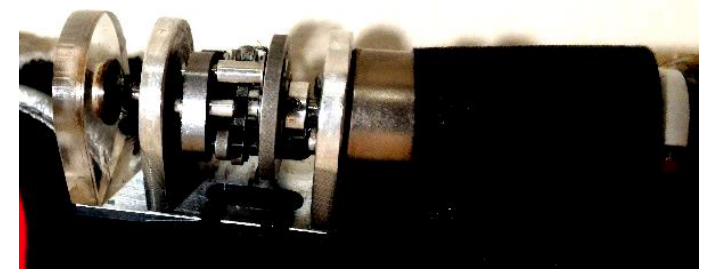

Figure 2. Hand grip assistive device clutch gearing system

In this paper, a new control system for the hand grip assist device was introduced. The control system takes advantages of the input from two sensors. The first sensor is electromyography (EMG) $[7,18,20]$. The EMG sensor was use to obtain EMG signal from the muscle. The second sensor is flex sensor [15]. This sensor was placed on top of finger. The function of flex sensor is as an indicator for finger during flexing and extending. Both signal from the sensors then feed into a microcontroller and gives output signal to control the actuator of the hand grip assistive device.

In the new control, the user can experienced two mode of usage in the same device. First mode is semi-active. In semi-active mode, the user will only need to move their hand a little bit and the device will support their gripping until the end. The second mode is known as active. When the user moves their hand, the device will support their gripping a little bit and user needs to move their hand again to make the device support more. Active and semiactive modes have advantages to promote user who suffer hand weakness to use their hand and not too depended on the device. On the safety sides, the device has built in safety switch for emergency stop. Besides that, the flex sensor also uses to prevent hyper flexing which safety program is built in the microcontroller. This measure was taken to prevent any accident that may occur to the user.

\section{Methodology}

\subsection{Control Hardware and Sensors}

First step of the device improvement was to implement sensors on the hand grip assistive device. This is to replace the used of manual switches that was implemented on the device previously. There are two type of sensor was used. First is an electromyography (EMG) sensor shown in Figure 3, which use to acquire EMG signal from muscle movement. In this research, the EMG sensor was used to acquire the EMG signal from Flexor Digitorum Profundus muscle that was mainly used to flexing fingers of hand.

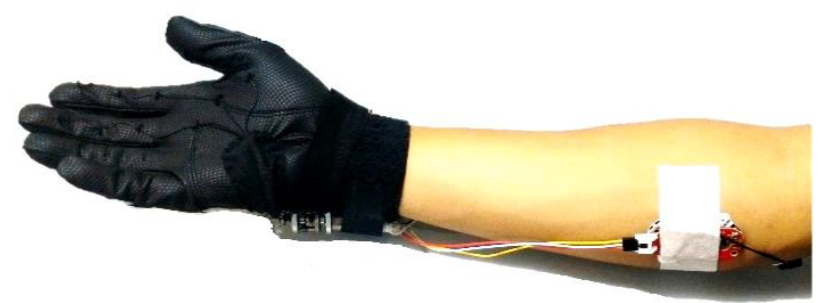

Figure 3. Electromyography sensor placement on the forearm

The second sensor is flex sensor as shown in Figure 4 that was used as indicator during the finger flexing. The sensor was placed on top of the finger, so that when the finger flex, the sensor also bend along with the finger which will give input signal to the microcontroller. The input signal of the flex sensor was depended of how much the sensor strip was bended.

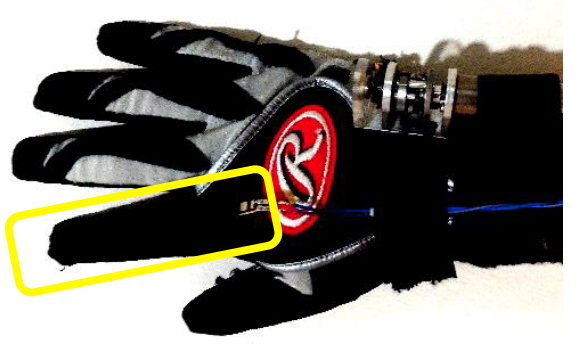

Figure 4. Flex sensor placement of the glove

The input signal from both sensors were sent to the microcontroller and the microcontroller will give out output signal to control the motor. The overall control flowchart is shown in Figure 5.

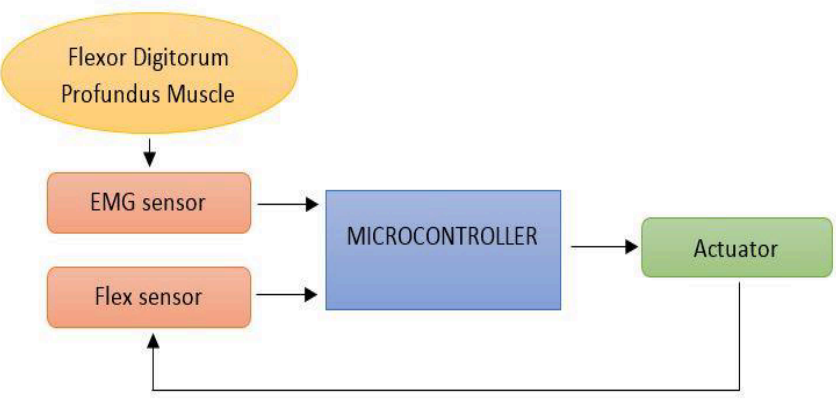

Figure 5. Overall flowchart for control hardware of hand Grip assistive device 


\subsection{Control Programming}

The control program for the hand grip assistive device consists of three parts. The first part of the program is threshold reading program and the flowchart of the program shown in Figure 6. The program initializes to set the threshold value for EMG signal. During the program, user needs to move their hand for several times in a time range that was set. The program will capture the average maximum and minimum value of the EMG signal. From the value, threshold for EMG signal was produced. The value was used in active and semi-active mode program.

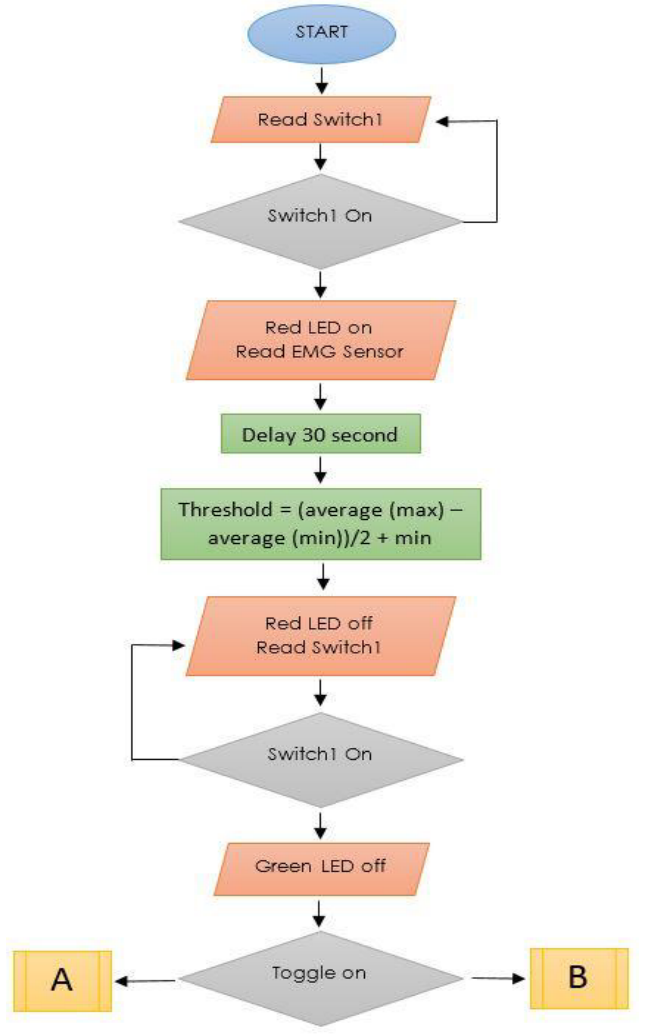

Figure 6. Threshold reading programming flowchart

The other two parts of program is mode program. There are two types of mode for the user using the hand grip assistive device which are semi-active and active mode. The semi-active mode program flowchart is shown in Figure 7. The semi-active mode start with the EMG sensor acquiring the EMG signal from the muscle and compared the obtain signal with the threshold value. If the comparison is larger, the microcontroller give out output signal to the motor and moved until the flex value maintain its value for a range of time before the motor stopped. The semi-active mode used single signal from EMG sensor to activate the device for gripping activities.

On the other hand, the active mode flowchart program shown in Figure 8. Instead of using single EMG signal to activate the device in semi-active mode, active mode required user to continuously feed the EMG signal to activate the hand grip assistive device. From Figure 8, the program consist of the iteration of acquiring EMG signal, compare it with the threshold value then motor moved in limited range. The iteration continuous until the flex value maintains its value for a range of time and the motor stopped.

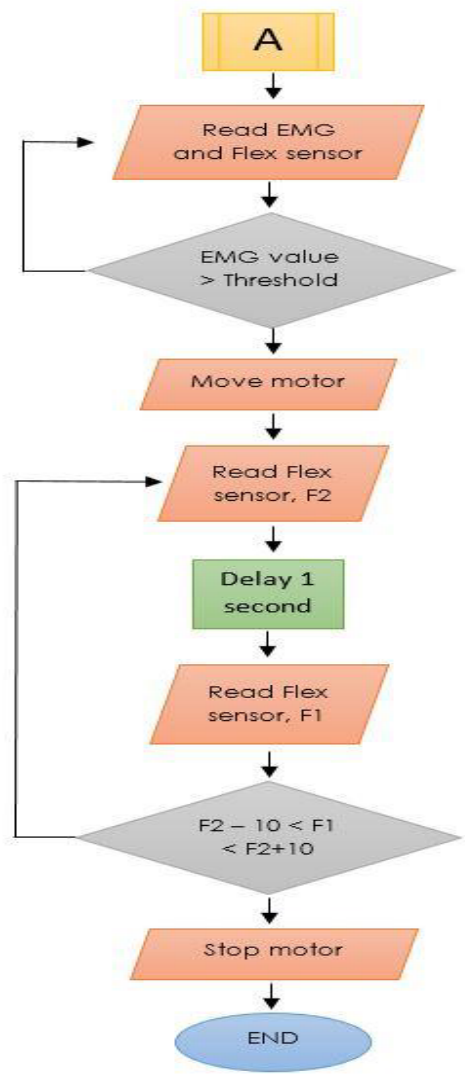

Figure 7. Semi-active mode programming flowchart

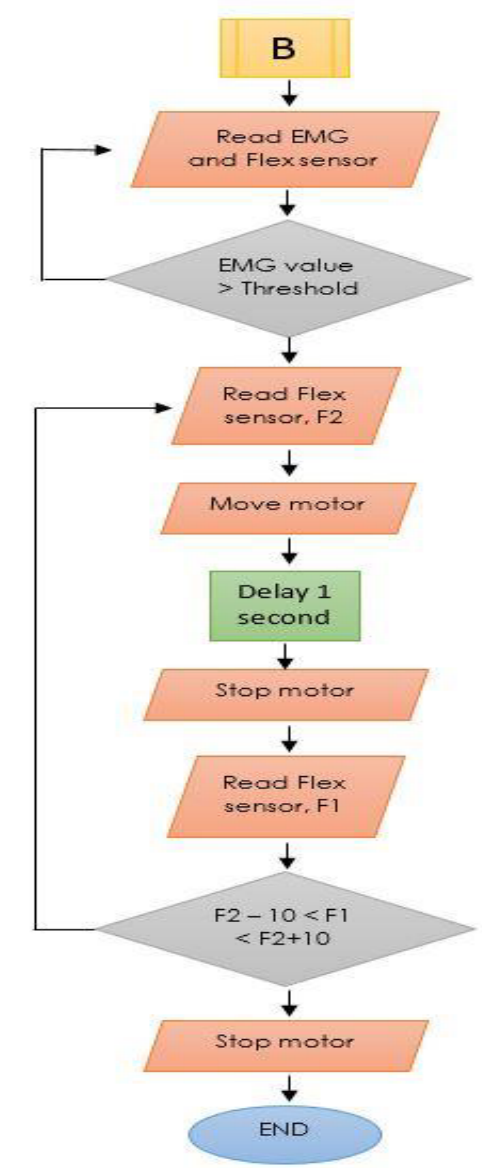

Figure 8. Active mode programming flowchart 


\subsection{Device Workability Test}

The test was conducted to verify the workability of the hand grip assistive device with the new control system. The test was conducted to ensure the safety of the device before experiment it with old people.

For the device workability test, one normal subject age 24 years was selected. The subject was required to do two tasks for each mode. The tasks were consist of gripping with empty hand and with cylindrical shape object. The objective of the tasks was to imitate the grip motion by hand. The subject also required to purposely use minimal strength in this test in order to imitate hand weakness of the old people. The test was carried out, and the flow of the test shown in the Figure 9.

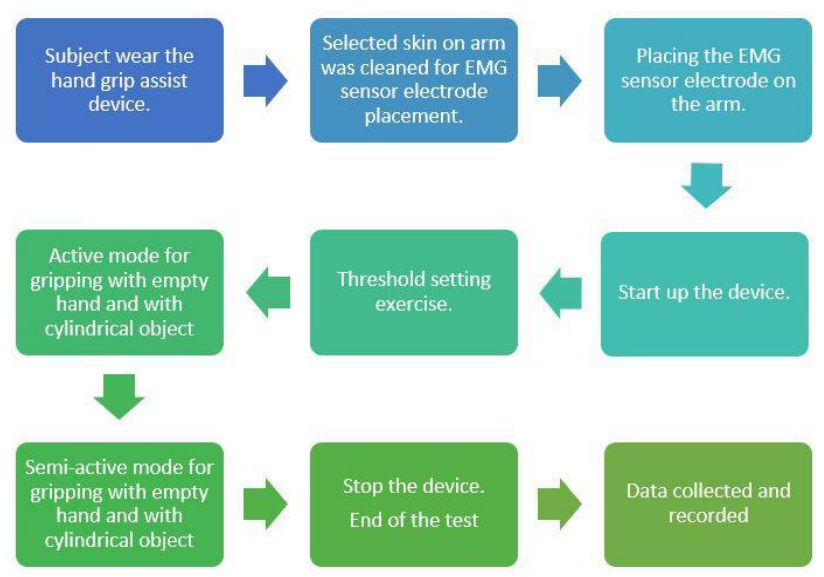

Figure 9. Hand grip assist device workability test flow

\section{Results and Discussion}

As the electromyography signal level always changing due to certain cause such as skin thickness, different placement of the electromyography sensor electrode, different user and many more, the auto calibrate threshold value was required. Thus, threshold calculation program was developed to produce the threshold value through setting exercise. Figure 10 shows the electromyography signal during threshold calculation step.

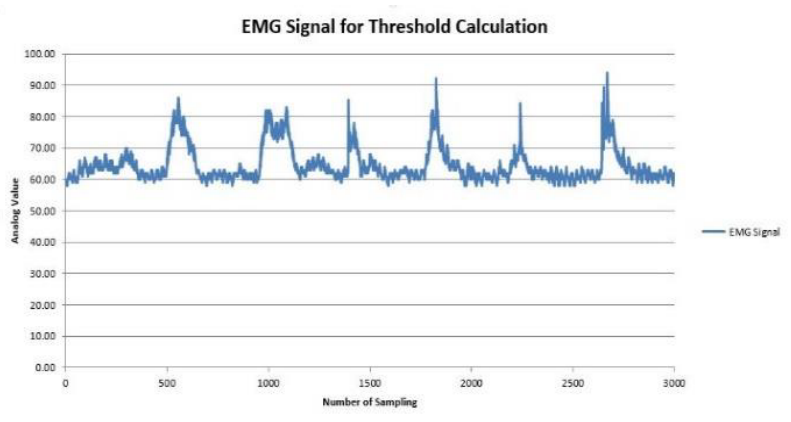

Figure 10. EMG signal during threshold calculation

During the exercise, the user was required to open and close their fingers for several in times in the time range that was set. Then, in the threshold calculation program, the electromyography signal was separated in minimum and maximum value for each 100 sets of reading. For 30 sets of the minimum and maximum value, average maximum and minimum value of the electromyography signal was obtain. The threshold value was obtained using simple formula below.

$$
\begin{gathered}
\text { Threshold Value }=((\text { Average Max }- \text { Average Min }) \div 2) \\
+ \text { Average Min }
\end{gathered}
$$

The threshold value is used for the semi active and active mode program. In semi active mode program, the electromyography signal will act as one time activator for the program. When the electromyography signal obtain greater than the threshold value, the program was activated. Figure 11 below shows the EMG and flex sensor signal obtain from the semi-active mode program.

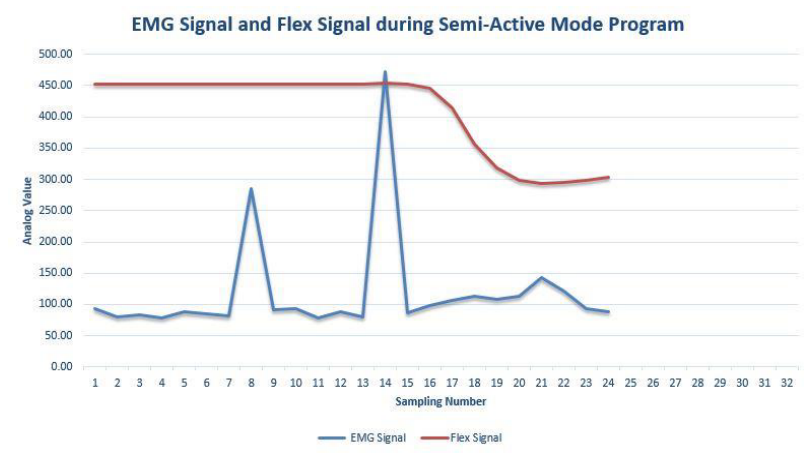

Figure 11. EMG and flex signal during semi-active program

From the figure, when the electromyography signal is high or exceeds the threshold value, the flex signal starts to decrease its value. The flex signal indicated that the finger is in flexing motion as the flex signal value is decrease. The flexion motion was produce by the activation of DC motor of the hand grip assist device. The motor continue to move until the flex signal was maintain at certain value. This happen due to that the flexion was reach to limit and then the motor stop to hold the flexion state.

In the active mode, the user was required to provide electromyography signal for several times to activate the DC motor on the hand grip assist device. When, user move their finger and electromyography signal was higher than threshold value, the motor move and flex the finger little bit and then stop. This step was repeated until the flexion reached to the limit. Figure 12 below shows the electromyography and flex signal during active mode program.

From the figure, it shows that when electromyography signal is high and exceed the threshold value, the flex signal decrease a little bit and maintain at that value. When another electromyography signal value exceeds the threshold value, the flex signal decreased and stops again. This cycle repeated until the flex signal value cannot decrease anymore which indicate that the limit was reached.

In both program, the flex sensor also act as limiter or stopper during gripping session. In the gripping motion, if the flex value constant at certain level, it stop the motor and release the gear to free up the cable. Beside that it 
also acts as safety indicator in the program that prevents hyper flexion of the finger.

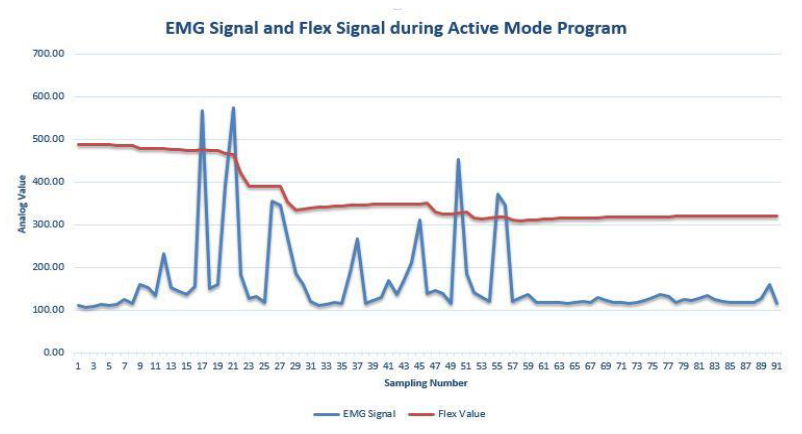

Figure 12. EMG and flex signal during active program

\section{Conclusion}

In conclusion, the hand grip assistive device is a device that is expected to support the old people in their gripping activities. The device is in a form of a wearable use to improve gripping power of old people. The new developed control system, implementing two sensors which electromyography and flex sensor. The control program consists three main parts that are threshold calculation program, semi active mode program and active mode program. The modes are for user to select which mode suitable for them. This new control program allow user to not over depended on the device and try to active the device by moving their own finger. It is hope that the new control system for the hand grip assist device able to improve user experience in using this device.

\section{Acknowledgement}

This research is funded by the Ministry of Education Malaysia under the Prototype Research Grant Scheme (PRGS) [Grant no: PRGS/TK01/(01)1167/2014(01)]. The authors would like to thank Universiti Malaysia Sarawak (UNIMAS) and Shibaura Institute of Technology, Japan for providing the facilities for this research.

\section{References}

1. World Health Organiztion (WHO), Health Statistic and Information Systems: Definition of an older or elderly people.

Retrieved from: http://www.who.int/healthinfo/ survey/ageingdefnolder/en/

Retrieved on: $20^{\text {th }}$ May 2016

2. World Population Ageing 2013. Department of Economic and Social Affairs Population Division, United Nation, New York.

3. United Nations Population Division. World Population Prospects: The 2012 Revision. Crude Birth Rate.

Retrieved from: http://data.un.org/Data.aspx?q= world + population $\& \mathrm{~d}=$ PopDiv $\& \mathrm{f}=$ variableID $\% 3 \mathrm{~A} 53$ $\% 3$ BcrID $\% 3$ A900
Retrieved on: $20^{\text {th }}$ May 2016

4. World Health Organization (WHO). Global Health Observatory (GHO) Data: Life Expectancy

Retrieve from: http://www.who.int/gho/ mortality_burden_disease/life_tables/situation_trends text/en/

$\bar{R}$ etrieve on: $20^{\text {th }}$ May 2016

5. P. Heo, G.M. Gu, S.J. Lee, K. Rhee and J. Kim, Current Hand Exoskeleton for Rehabilitation and Assistive Engineering, International Journal of Precision Engineering and Manufacturing. Vol. 13, No. 5, pp. 807-824 (2012)

6. S. Ueki, H. Kawasaki, S. Ito, Y. Nishimoto, M. Abe, T. Aoki, Y. Ishigure, T. Ojika, and T. Mouri, Development of a Hand-Assist Robot with MultiDegrees-of-Freedom for Rehabilitation Therapy, IEEE/ASME Transactions on Mechatronics, Vol. 17, No. 1 (2012)

7. N.S.K. Ho, K.Y. Tong, X.L. Hu, K.L. Fung, X.J. Wei, W. Rong and E.A. Susanto, An EMG-driven Exoskeleton Hand Robotic Training Device on Chronic Stroke Subjects, 2011 IEEE International Conference on Rehabilitation Robotics (2011)

8. Y.L. Fu, Q.C. Zhang, F.H. Zhang and Z.K. Gan, Design and Development of a Hand Rehabilitation Robot for Patient-Cooperative Therapy Following Stroke, Proceedings of the 2011 IEEE International Conference on Mechatronics and Automation (2011)

9. A. Chiri, N. Vitiello, F. Giovacchini, S. Roccella, F. Vecchi, and M. C. Carozza, Mechatronic Design and Characterization of the Index Finger Module of a Hand Exoskeleton for Post-Stroke Rehabilitation, IEEE/ASME Transactions on Mechatronics. Vol. 17, No. 5 (2012)

10. M. Cempini, S.M.M.D. Rossi, T. Lenzi, M. Cortese, F. Giovacchini, N. Vitiello and M.C. Carozza, Kinematics and Design of a Portable and Wearable Exoskeleton for Hand Rehabilitation, 2013 IEEE International Conference on Rehabilitation Robotics (2013)

11. J. Iqbal, H. Khan, N.G. Tsagarakis, and D.G. Caldwell, A Novel Exoskeleton Robotic System for Hand Rehabilitation - Conceptualization to Prototyping, Biocybernatics and Biomedical Engineering. Vol 34, pp 79-89 (2014)

12. H.K. In, K.J. Cho, K.R. Kim and B.S. Lee, Jointless Structure and Under-Actuation Mechanism for Compact Hand Exoskeleton, 2011 IEEE International Conference on Rehabilitation Robotics (2011)

13. E.B. Brokaw, I. Black, R.K. Holley, and P.S. Lum, Hand Spring Operated Movement Enhancer (HandSOME): A Portable, Passive Hand Exoskeleton for Stroke Rehabilitation, IEEE Transactions on Neural Systems and Rehabilitation Engineering. Vol. 19, No. 4 (2011)

14. P. Polygerinos, Z. Wang, K. C. Galloway, R.J. Wood, and C.J. Walsh, Soft Robotic Glove for Combined Assistance and at-Home Rehabilitation, Robotics and Autonomous Systems. Vol 73, pp. 135143 (2015) 
15. J.H. Bae, Y.M. Kim and I. Moon, Wearable Hand Rehabilitation Robot Capable of Hand Function Assistance in Stroke Survivors, The Fourth IEEE RAS/EMBS International Conference on Biomedical Robotics and Biomechatronics (2012)

16. H.K. In, B.B.H. Kang, M.K. Sin and K.J. Cho, ExoGlove: A Wearable Robot for the Hand with a Soft Tendon Routing System, IEEE Robotics and Automation Magazine. Pp. 97-105 (2015)

17. K. Toya, T. Miyagawa and Y. Kubota, Power Assist Glove Operated by Predicting the Grasping Mode, Journal of System Design and Dynamics. Vol. 5, No. 1, pp.94-108 (2011)

18. K. Tadano, M. Akai, K. Kadota and K. Kawashima. Development of Grip Amplified Glove using Biarticular Mechanism with Pneumatic Artificial Rubber Muscle, 2010 IEEE International Conference on Robotics and Automation (2010)

19. Y. Hasegawa, J. Tokita, K. Kamibayashi and Y. Sankai, Evaluation of fingertip force accuracy in different support conditions of exoskeleton, 2011 IEEE International Conference on Robotics and Automation (2011)

20. H.J. Lee, K.H. Kim and S.R. Oh, Development of a Wearable and Dry sEMG Electrode System for Decoding of Human Hand Configurations, 2012 IEEE/RSJ International Conference on Intelligent Robots and Systems (2012) 